\title{
Diferencias en las perspectivas discursivas sobre la vejez y la muerte en adultos mayores pertenecientes a dos instituciones geriátricas de Bucaramanga
}

Differences in discursive perspectives on old age and death in the elderly who belong to two nursing homes in Bucaramanga

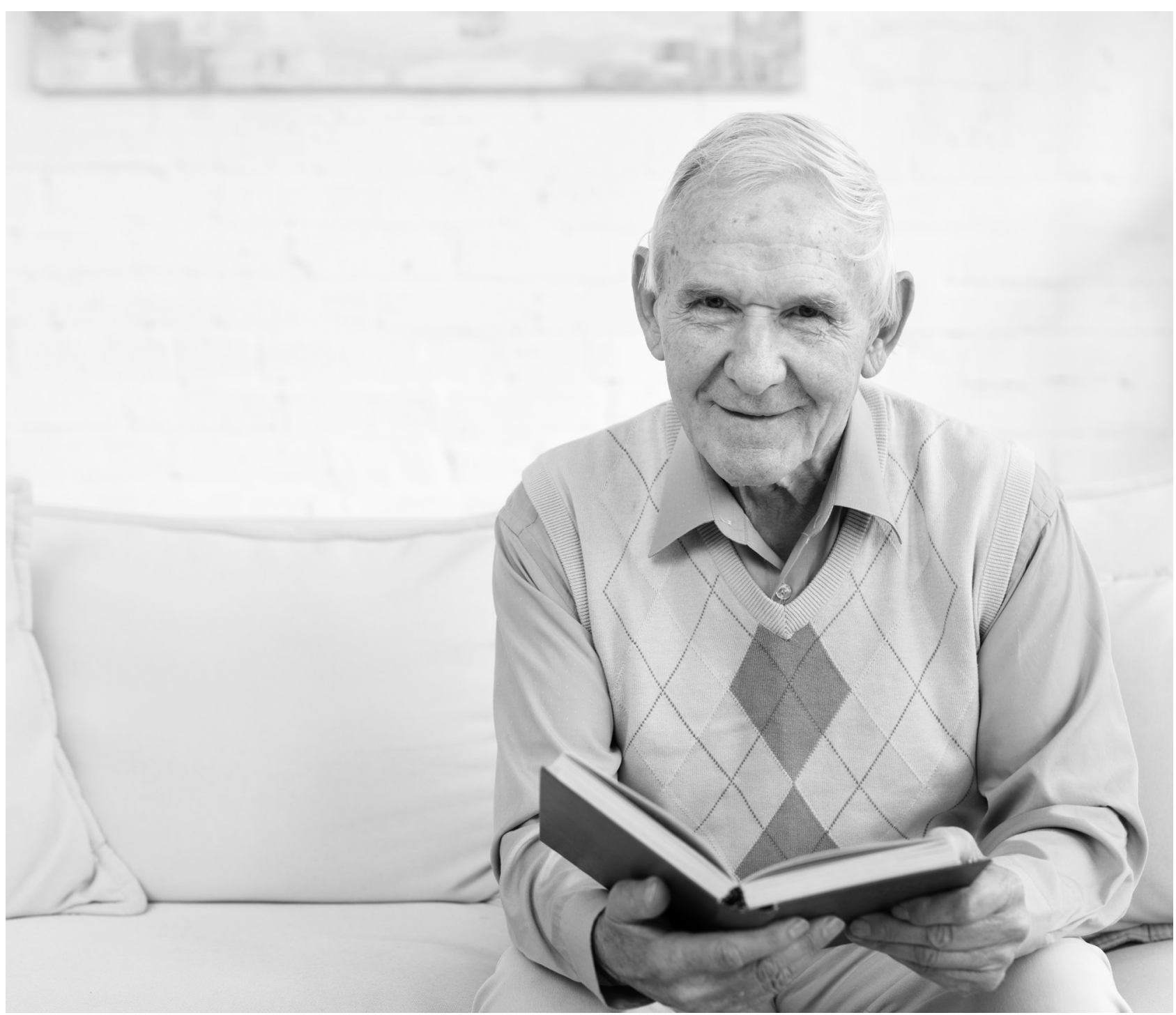




\title{
Diferencias en las perspectivas discursivas sobre la vejez y la muerte en adultos mayores pertenecientes a dos instituciones geriátricas de Bucaramanga ${ }^{1}$

\author{
Differences in discursive perspectives on old age and death in the \\ elderly who belong to two nursing homes in Bucaramanga
}

\author{
Laura Juliana Barchelot Aceros ${ }^{2}$, Lina Katherine Valencia Silva ${ }^{3}$, Suzette Tatiana \\ Poveda Esteban ${ }^{4}$, Emily Carolina González Clemente ${ }^{5}$
}

Artículo recibido en marzo 2 de 2020; artículo aceptado en mayo 28 de 2020

\begin{abstract}
Este artículo puede compartirse bajo la Licencia Creative Commons Atribución-NoComercial-Compartirlgual 4.0 Internacional y se referencia usando el siguiente formato: Barchelot, L. J., Valencia, L. K., Poveda, S. T. y González, E. C. (2020). Diferencias en las perspectivas discursivas sobre la vejez y la muerte en adultos mayores pertenecientes a dos instituciones geriátricas de Bucaramanga. I+D Revista de Investigaciones, 15 (2), 58-69. DOI: https://doi.org/10.33304/revinv.v15n2-2020006
\end{abstract}

\begin{abstract}
Resumen
La presente investigación tuvo como objetivo principal comparar las perspectivas discursivas acerca de la vejez y la muerte en adultos mayores pertenecientes a dos instituciones geriátricas (una pública y una privada) de Bucaramanga. Para ello se contó con la participación de diez adultos mayores, a quienes se les practicó una entrevista semiestructurada. Se organizó y clasificó la información obtenida mediante el programa ATLAS ti (v.7.5), y se generó la construcción de categorías y subcategorías que permitieron evidenciar, en el Geriátrico Público, síntomas depresivos en relación con la vejez, así como pensamientos o ideaciones suicidas respecto a la muerte, a diferencia del Geriátrico Privado, donde prevalece una perspectiva positiva frente a la vejez y una postura religiosa frente a la muerte. Finalmente, se buscó brindar un aporte científico en el ámbito regional, ya que son pocos los estudios realizados con esta población, y fomentar la implementación de planes de promoción y prevención dirigidos a la institución.
\end{abstract}

Palabras clave: Adultos mayores, envejecimiento, institución geriátrica, muerte, perspectivas discursivas, vejez.

\footnotetext{
${ }^{1}$ Tipo de artículo empírico de enfoque cualitativo, resultado de un proyecto de investigación culminado, perteneciente al área de ciencias sociales, subárea de psicología, desarrollado en apoyo con el Grupo de Investigación UDIPSI, Universidad de Investigación y desarrollo UDI (Bucaramanga, Colombia). Dirección: calle 9 n. 23-55, PBX: 6352525. Fecha de inicio: 1 de agosto de 2018. Fecha de terminación: 2 de julio de 2019.

${ }^{2}$ Magíster en Investigación Clínica, Universidad de Guanajuato. Docente investigadora vinculada al Grupo de Investigación UDIPSI, de la Universidad de Investigación y Desarrollo (Bucaramanga, Colombia). Dirección: calle 9 n. 23-55. PBX: 6352525. ORCID ID: https://orcid.org/0000-0002-47763721. Correo electrónico institucional: Ibarchelot1@udi.edu.co.

${ }^{3}$ Psicóloga, Universidad de Investigación y Desarrollo (UDI) (Bucaramanga, Colombia). Vinculada en la empresa Ingeniería y Construcciones MH SAS (Floridablanca, Colombia). Dirección: calle 40 n. 5-53, PBX: 6424290. ORCID ID: https://orcid.org/0000-0002-9469-0596. Correo electrónico institucional: Ivalencia2@udi.edu.co

${ }^{4}$ Psicóloga, Universidad de Investigación y Desarrollo (UDI) (Bucaramanga, Colombia). Vinculada en la empresa Ingeniería y Construcciones MH SAS (Floridablanca, Colombia). Dirección: calle 40 n. 5-53, PBX: 6424290. ORCID ID: https://orcid.org/0000-0002-7986-6039. Correo electrónico institucional: Jpoveda1@udi.edu.co.

${ }^{5}$ Magíster en Psicología Clínica y de la Salud, Universidad Central de Venezuela. Docente de Universidad de Investigación y Desarrollo (UDI) (Bucaramanga, Colombia). Dirección: calle 9 n. 23-55, PBX: 6352525. ORCID ID: https://orcid.org/0000-0001-7472-0789. Correo electrónico institucional: egonzalez5@udi.edu.co.
} 
Laura Juliana Barchelot Aceros, Lina Katherine Valencia Silva, Suzette Tatiana Poveda Esteban, Emily Carolina González Clemente Diferencias en las perspectivas discursivas sobre la vejez y la muerte en adultos mayores pertenecientes a dos instituciones geriátricas de Bucaramanga

\section{Abstract}

The main objective of this research is to compare the discursive perspectives of old age and death in elderly from two nursing homes (one public and one private) of Bucaramanga. Ten older adults participated in the study, with a semi structured interview. The information obtained through the ATLAS ti program (v.7.5) was organized and classified, which generated the construction of categories and subcategories that allowed to evidence depressive symptoms in relation to old age and suicidal thoughts with respect to death in the public nursing home, unlike the private one, where there is a positive perspective about old age and a religious position towards death prevails. Finally, it was sought to provide a scientific contribution at the regional level, since there are few studies carried out on this population, and to promote the implementation of promotion and prevention plans directed at the institution.

Keywords: Elderly, aging, geriatric, nursing home, death, discursive perspectives, old age.

\section{Introducción}

Esta investigación surge como una necesidad apremiante ante el envejecimiento progresivo de la población adulta mayor. De acuerdo con la Organización Mundial de La Salud (OMS, 2017), se estima que entre el año 2015 y el 2050 la población mayor de 60 años se multiplicará casi por dos, debido al envejecimiento acelerado de la población mundial, producto del incremento de la esperanza de vida poblacional. En ese sentido, el Ministerio de Salud y Protección Social (2013) ha destacado cómo en Colombia las cifras de envejecimiento poblacional también van en aumento. En el año 2010 el porcentaje de envejecimiento fue de $34,4 \%$; en el año 2015, de 41,5\%, y para el año 2020 se espera un incremento del envejecimiento poblacional del $49,8 \%$. Es esta una realidad de la cual no se escapa el departamento de Santander, que ocupa el sexto puesto de treinta departamentos analizados, con mayor índice de envejecimiento poblacional, con lo que constituye un $42 \%$. Esto conduce a importantes desafíos sociales, si se considera el cambio individual, familiar y social que atraviesa esta población.

Por ende, y pretendiendo acercarnos a esta realidad, se hace necesario el desarrollo de estudios investigativos que se centren en conocer la perspectiva discursiva del adulto mayor frente a la vejez y la muerte, y los aspectos sociodemográficos más relevantes que pueden influir en la percepción de cada geronte. Dicha necesidad surge ante la ausencia de apoyo social, producto de la indiferencia de la sociedad y del entorno cercano del adulto mayor, como familia, amigos, vecinos, etc. De esta manera lo han destacado Cardona-Arango et al. (2010), quienes manifiestan cómo los principales motivos de institucionalización del adulto mayor son el abandono ante el desamparo y la vulnerabilidad social, y la decisión familiar, acompañada de humillaciones por parte de los seres queridos. Gran parte de los adultos mayores terminan siendo institucionalizados en hogares geriátricos, a causa de los pocos recursos económicos y la marginación social (Aranda et al., 2001).
Teniendo en cuenta lo anterior, es necesario entender la muerte como parte del proceso del ciclo vital del ser humano. Ante esto, Rubio (1981) y Rivera y Mancinas (2007) destacan cómo el concepto de muerte va sufriendo transformaciones según el momento evolutivo en el que se encuentre un individuo: se inclina hacia la aceptación en las últimas etapas de vida, y se llega a considerar la muerte como una sucesión natural e inevitable.

El envejecimiento implica un proceso que acarrea una serie de transformaciones fisiológicas, sociales, psicológicas y funcionales que se dan a lo largo de todo el ciclo vital. Por su parte, la vejez hace referencia a la última etapa de este proceso. No obstante, el estudio de la vejez debe contemplar la comprensión del envejecimiento, ya que las características de cada geronte serán consecuencia de las experiencias que han marcado todo el proceso vital, si se tiene en cuenta la complejidad de ambos fenómenos, que incluyen dimensiones individuales, sociales y biológicas interdependientes (Dulcey, 2015; López y Marín, 2016; Santos y Valencia, 2015).

Ante la llegada de la vejez, se suele incrementar la conciencia de finitud de la vida, lo que provoca en el adulto mayor un acto reflexivo sobre el presente y el futuro a modo de revisión de sus propias experiencias. Se permite la valoración de las decisiones tomadas en el pasado, y se plantea la resolución de conflictos pendientes en el presente (Dulcey, 2015). Desde la perspectiva de la teoría psicosocial de Erikson (1982), y de acuerdo con Río et al. (2014), la vejez se entiende como el último periodo de una secuencia de etapas normativas predeterminadas. En este, el individuo debería contar con una mayor identidad, participación y compromiso social, pues debió ir adquiriendo esto a lo largo de toda su vida. Por lo tanto, para Seelbach (2013), esta etapa de la vida constituye el estadio con mayor relevancia personal y social, ya que permite la trascendencia en el nivel de la autorrealización del individuo.

Por lo tanto, se toma el envejecimiento y la 
institucionalización como parte de la perspectiva discursiva del adulto mayor. Estos conceptos se asocian con la vejez y la muerte como aspecto de este proceso investigativo, que tiene como objetivo principal el comparar las perspectivas discursivas de los adultos mayores institucionalizados de dos hogares geriátricos (uno público y uno privado) de Bucaramanga en relación con estos dos tópicos: "vejez" y "muerte". Por esta razón, se planteó la siguiente pregunta de investigación: ¿Existen diferencias en las perspectivas discursivas sobre la vejez y la muerte en adultos mayores de una institución geriátrica pública y una privada de Bucaramanga?

\section{Metodología}

\section{Tipo de estudio}

Esta investigación se desarrolla bajo un enfoque cualitativo, en el que, de acuerdo con Hernández, Fernández y Baptista (2014), se busca "comprender los fenómenos, explorándolos desde la perspectiva de los participantes en un ambiente natural y en relación con su contexto" (p. 358). Se realizó mediante un diseño fenomenológico, con el fin de estudiar la experiencia vital desde la cotidianidad, y en atención a las perspectivas de los participantes desde el estudio de los significados que dan a la vejez y la muerte (Rodríguez et al., 1999). A través de sus relatos, se realizó un análisis de contenido. Mediante la triangulación de las categorías teóricas propuesta y las categorías emergentes extraídas de las experiencias de los informantes, se interpretaron sus narrativas en relación con sus características sociodemográficas, y se consideró el hogar geriátrico (público o privado) en el que reside.

\section{Participantes}

Se seleccionó una muestra por conveniencia, conformada por 10 participantes, cuyas edades oscilaron entre 65 y 95 años, subdivididos en 5 adultos mayores de una institución geriátrica de carácter privado, "Hogar Geriátrico Alegría e llusiones de Vivir", y 5 adultos mayores pertenecientes a una institución geriátrica de carácter público, "Hogar Jerusalén", de la ciudad de Bucaramanga.

De igual forma, se tuvieron en cuenta para la inclusión de los participantes en el estudio los siguientes criterios: 1) pertenecer a un hogar geriátrico público o privado del área metropolitana de Bucaramanga; 2) estar de acuerdo con la participación en la investigación; 3) estar en el rango de edad de 65 a 95 años; 4) contar con una antigüedad mínima de seis meses dentro del hogar geriátrico y 5) no presentar algún tipo de limitación auditiva y/o del habla. A su vez, se establecieron como criterios de exclusión 1) que el adulto mayor estuviera en internamiento parcial; 2) la presencia de algún tipo de disfunción que limite el habla y/o audición y (3) que el adulto no deseara participar de la investigación.

Por último, como criterios de eliminación: 1) adultos mayores que durante la aplicación de la entrevista se negaran a terminar de contestarla; (2) adultos mayores que una vez confirmada su participación en la investigación, por circunstancias externas, ya no se encontraran en la institución.

\section{Materiales e instrumentos}

\section{Entrevista semiestructurada}

Se empleó como instrumento principal la entrevista semiestructurada, pues es una técnica que se apoya en el enfoque y diseño de la investigación cualitativa, que, de acuerdo con Vargas-Jiménez (2012), conduce a la interacción entre el entrevistado y entrevistador, en aras de adentrarse a la intimidad de los participantes y poder entender desde la propia individualidad la realidad del fenómeno abordado. De esta manera se accedió a las perspectivas de vejez y muerte en el discurso del adulto mayor, mediante sus relatos y experiencias.

Sumado a ello, y con el fin de garantizar la rigurosidad teórica y metodológica de la entrevista semiestructurada creada por las autoras del trabajo, el instrumento fue sometido a revisión por parte de dos figuras expertas en el campo de la gerontología y de la metodología investigativa.

Cabe resaltar que con la entrevista semiestructurada se abarcaron cuatro (4) subcategorías de análisis (vejez, muerte, envejecimiento e institucionalización), a partir de la categoría de perspectivas discursivas. Adicionalmente, se recopiló información sobre las características sociodemográficas de la muestra de estudio.

\section{Ficha de datos sociodemográficos}

Se aplicó una ficha de datos sociodemográficos, en la que se tuvieron en cuenta características como edad, sexo, estado civil, escolaridad, estado de salud, profesión u ocupación que ejercieron los adultos mayores durante su etapa vital previa a su jubilación. De igual forma, variables referentes al contexto en el que están, como la ubicación de la institución u hogar geriátrico y el estrato de este. Estos son factores que pueden influir en la percepción que el adulto mayor tiene respecto a la vejez y la muerte.

\section{Programa ATLAS ti (v.7.5)}

Teniendo en cuenta el enfoque cualitativo de la investigación, se hizo necesario el uso de la técnica de análisis de contenido y del programa sistematizado ATLAS ti, que permitió organizar y clasificar la información 
Laura Juliana Barchelot Aceros, Lina Katherine Valencia Silva, Suzette Tatiana Poveda Esteban, Emily Carolina González Clemente Diferencias en las perspectivas discursivas sobre la vejez y la muerte en adultos mayores pertenecientes a dos instituciones geriátricas de Bucaramanga

recolectada por medio de la entrevista semiestructurada, con lo que se garantizó un análisis y construcción de la información mediante las categorías que permitieron su adecuada interpretación. Estas fueron clasificadas de la siguiente manera: categorías (Perspectiva discursivas y Características sociodemográficas) y subcategorías (Envejecimiento, Vejez, Muerte e Institución). Sobre estas surgió una serie de categorías emergentes en relación con las preguntas planteadas y el discurso del adulto mayor.

\section{Observación natural}

Se realizó la investigación en el Hogar Geriátrico Alegría e Ilusiones de Vivir (ver Figura 1), institución de carácter privado de Bucaramanga, enfocada en ayudar con respeto, cariño e igualdad al adulto mayor para su cuidado y rehabilitación. Actualmente, el hogar geriátrico cuenta con 13 adultos mayores (6 hombres y 7 mujeres), con institucionalización total, a quienes se les brinda servicios de enfermería, psicología, nutrición y fisioterapia.

Las condiciones de infraestructura del hogar son adecuadas. Cuenta con un espacio considerable, suficientemente amplio para permitir el desplazamiento y ejecución de actividades ocupacionales. Así mismo, las habitaciones pueden ser dúplex (compartidas) o individuales, y están adaptadas a las condiciones de movilidad de cada adulto mayor, lo que les proporciona mayor comodidad.

Así mismo, se llevó a cabo la investigación en el Hogar Geriátrico Jerusalén (ver Figura 2), que cumple con características de una institución pública, debido a la infraestructura de la planta física y la carencia de servicios integrales para el acompañamiento del adulto mayor. Esta institución fue fundada hace trece años, y se enfoca en el cuidado y mejoramiento de las condiciones de vida del adulto mayor. Se sostiene mediante donaciones y aportes de familiares de los adultos institucionalizados. Actualmente, el hogar geriátrico cuenta con 22 adultos mayores (12 hombres y 10 mujeres), cinco de ellos diagnosticados con trastorno de bipolaridad y deterioro cognitivo.

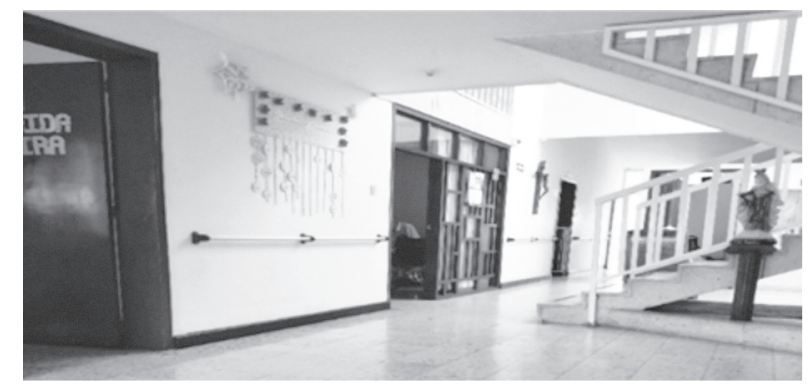

Figura 1. Instalación geriátrica privada. Fuente: Autoras. Nota: barandas ubicadas estratégicamente, antideslizantes en las escaleras para aquellos adultos mayores que se encuentran en la segunda planta, e iluminación adecuada, señalización y ambientación religiosa.

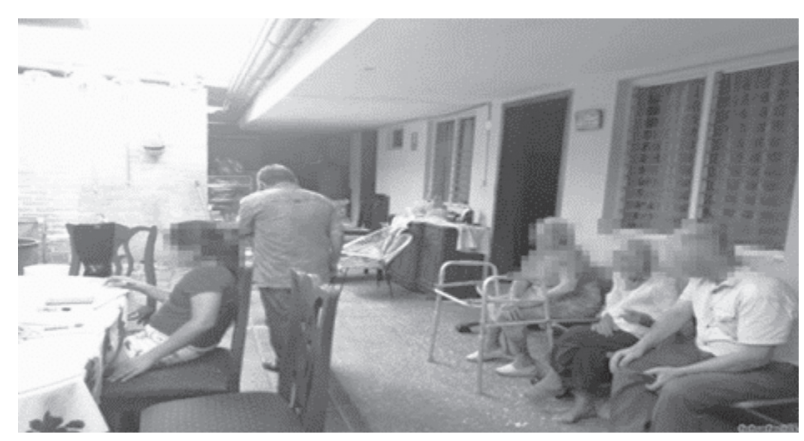

Figura 2. Instalación geriátrico público Fuente: Autoras. Nota: Comedor, sala o espacio de encuentro, habitaciones.

\section{Procedimiento}

Se contactó con las instituciones geriátricas, se socializaron los fines de la elaboración de esta investigación y se obtuvo el aval para su desarrollo. Posteriormente, se generó el contacto directo con la muestra de estudio, y se inició el proceso de captura de la información, previa socialización y firma del consentimiento informado por parte de los participantes. Se procedió entonces a indagar las características sociodemográficas, y finalmente se realizaron las entrevistas de manera individual con cada uno de los participantes.

Se resalta que las entrevistas fueron grabadas, para garantizar una exploración minuciosa del discurso del adulto mayor, así como para la transcripción de este, a fin de organizar y clasificar, a través del programa ATLAS ti, la información que de allí se desprenda.

\section{Resultados}

Los resultados de la investigación se obtuvieron mediante la triangulación de las categorías (ver Tabla 1) a partir de las teorías propuestas, las subcategorías y categorías emergentes que surgieron de las entrevistas, y que se ven reflejadas en la red sistemática de la información.

\section{Caracterización sociodemográfica}

Se considera importante la caracterización sociodemográfica en relación con la vejez, dado que, de acuerdo con el Ministerio de Salud y Protección Social (2018), esta representa una construcción social y biográfica de la vida del ser humano. Además, se enlaza con aspectos como edad, sexo, lugar de residencia, etc., que influyen en la percepción que el adulto mayor tiene de sí mismo y que se refleja en su discurso y actitud.

En la Tabla 2 se evidencia que las edades de los adultos mayores entrevistados en el Hogar Geriátrico Alegría e llusiones de Vivir (privado) oscilan entre los 71 y los 
95 años. Se encuentran estos dentro del rango de edad estipulado en la investigación (65-95 años), y la edad de 86 años es la que prevalece en los participantes. Cabe añadir que la mayoría de los adultos entrevistados fueron mujeres. Respecto al estado civil, se puede observar que prevalece el viudo y el soltero sobre el divorciado, y en cuanto al nivel de escolaridad, se encontró que no todos lograron culminar el proceso académico: el máximo nivel cursado por dos de los participantes fue la primaria, seguido del bachillerato, asociado a la ocupación u oficio (amas de casa) y profesión que sirvieron como base para que se desempeñaran en oficios como la asesoría de seguros, y en profesiones como la docencia de idiomas esto último en el caso de las mujeres-. Por otro lado, en lo que respecta al sexo masculino, estos participantes culminaron el proceso educativo con éxito, pues se alcanzó un nivel profesional (locutor Caracol). De igual forma, se denotó que las patologías más comunes en la muestra de estudio están relacionadas con enfermedades reumáticas (artrosis y osteoporosis).

Por otra parte, en el Hogar Jerusalén (público), las edades de los adultos mayores entrevistados oscilan entre los 65 y los 86 años. Estas edades son representativas de una muestra de 5 adultos mayores (una mujer y 3 hombres), sobre los que prevalece la edad de 65 años. Respecto al estado civil, se denota el casado o el divorciado sobre el soltero o el viudo. En cuanto al nivel de escolaridad, en contraste con el hogar geriátrico privado, se evidencia que solo cuatro de los cinco adultos mayores entrevistados cursaron la primaria, sin lograr culminarla con éxito, y uno alcanzó un título técnico, por lo que la mayoría se desempeñó en labores de agricultura. Por otro lado, solo dos participantes mencionaron padecer de alguna molestia física (hipertensión y Parkinson). Los restantes mantienen una condición de salud favorable.

Tabla 1

Triangulación de las categorías

\begin{tabular}{|c|c|c|c|c|c|}
\hline Categoría & Autores & Subcategoría & Autores & Categorías emergentes & Instrumento \\
\hline \multirow{3}{*}{$\begin{array}{l}\text { Perspectivas } \\
\text { discursivas }\end{array}$} & Garay et al. (2005) & Envejecimiento & $\begin{array}{l}\text { Ollar (1997) } \\
\text { citado en López } \\
\text { (2009) }\end{array}$ & $\begin{array}{l}\text { Aspectos + } \\
\text { Cambios físicos } \\
\text { Hobbies } \\
\text { Cambios emocionales } \\
\text { Espiritualidad } \\
\text { Relación familiar }\end{array}$ & \multirow{3}{*}{$\begin{array}{c}\text { Entrevista } \\
\text { semiestructurada }\end{array}$} \\
\hline & $\begin{array}{l}\text { Edwards (1997) } \\
\text { citado en Garay et } \\
\text { al. (2005) }\end{array}$ & Vejez & $\begin{array}{l}\text { Ministerio de } \\
\text { Salud y } \\
\text { Protección Social } \\
(2018) \\
\end{array}$ & $\begin{array}{l}\text { Edad } \\
\text { Actitud } \\
\text { Dependencia } \\
\text { Enfermedades } \\
\end{array}$ & \\
\hline & Gergen (2007) & Muerte & $\begin{array}{l}\text { Serra y } \\
\text { Abengózar } \\
(1990)\end{array}$ & $\begin{array}{l}\text { Aceptación } \\
\text { Miedo } \\
\text { Postura religiosa } \\
\text { Experiencia cercana } \\
\text { Imaginario de muerte } \\
\end{array}$ & \\
\hline \multirow{2}{*}{$\begin{array}{c}\text { Características } \\
\text { sociodemográficas }\end{array}$} & $\begin{array}{l}\text { Garrido y Álvaro } \\
\text { (2007) }\end{array}$ & \multirow{2}{*}{ Institución } & $\begin{array}{l}\text { Zavala et al. } \\
\text { (2006) }\end{array}$ & \multirow{2}{*}{$\begin{array}{l}\text { Motivo de } \\
\text { institucionalización } \\
\text { Dinámica institucional } \\
\text { Relaciones sociales } \\
\text { Trato del cuidador }\end{array}$} & \multirow{2}{*}{$\begin{array}{c}\text { Ficha } \\
\text { sociodemográfica } \\
\text { Entrevista }\end{array}$} \\
\hline & $\begin{array}{l}\text { Gergen (1999) } \\
\text { citado en Garrido } \\
\text { y Álvaro (2007) }\end{array}$ & & Barenys (2012) & & \\
\hline
\end{tabular}

Fuente: Autoras.

Tabla 2

Caracterización sociodemográfica de los participantes

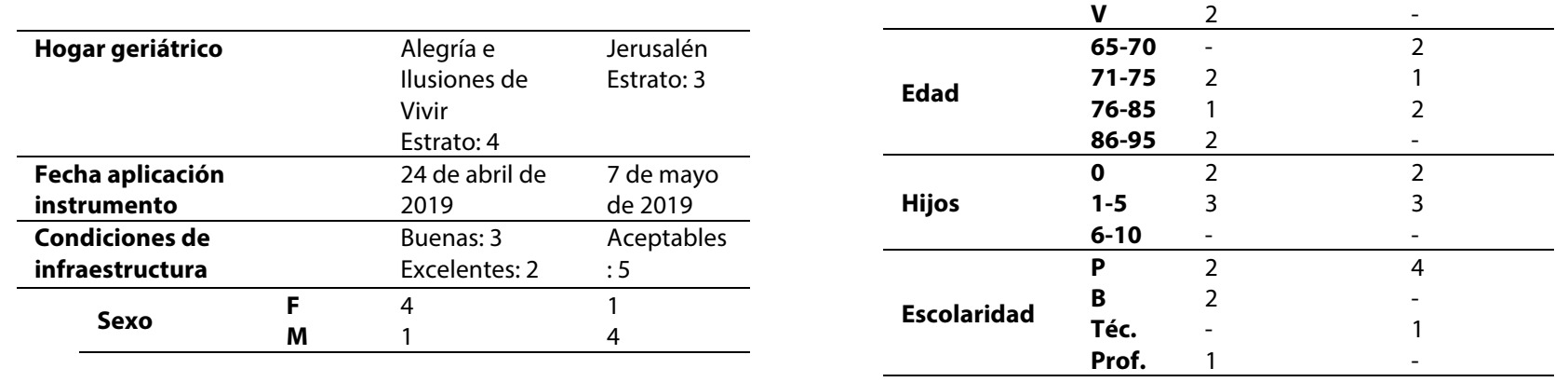


Laura Juliana Barchelot Aceros, Lina Katherine Valencia Silva, Suzette Tatiana Poveda Esteban, Emily Carolina González Clemente Diferencias en las perspectivas discursivas sobre la vejez y la muerte en adultos mayores pertenecientes a dos instituciones geriátricas de Bucaramanga

\begin{tabular}{|c|c|c|c|}
\hline \multicolumn{2}{|c|}{ Profesión ejercida } & $\begin{array}{l}\text { Ama de casa: } 2 \\
\text { Asesoría de } \\
\text { seguros: } 1 \\
\text { Docencia: } 1 \\
\text { Locutor: } 1\end{array}$ & $\begin{array}{l}\text { Agricultor: } \\
3 \\
\text { Niñera: } 1 \\
\text { Tecnólogo } \\
\text { en } \\
\text { Mercadeo } \\
\text { y Ventas: } 1\end{array}$ \\
\hline \multicolumn{2}{|c|}{$\begin{array}{l}\text { Enfermedad o } \\
\text { problema de salud }\end{array}$} & $\begin{array}{l}\text { Artrosis: } 1 \\
\text { Artrosis- } \\
\text { Epilepsia: } 1 \\
\text { Artrosis - } \\
\text { osteoporosis: } 1 \\
\text { Paraplejia: } 1 \\
\text { Poliomielitis: } 1\end{array}$ & $\begin{array}{l}\text { Artrosis: } 0 \\
\text { Hipertensió } \\
\text { n: } 1 \\
\text { Parkinson: } \\
1\end{array}$ \\
\hline $\begin{array}{l}\text { Tiempo } \\
\text { institucionali } \\
\text { zado }\end{array}$ & $\begin{array}{l}\text { 8-11 } \\
\text { meses } \\
1-4 \\
\text { años } \\
5-8 \\
\text { años }\end{array}$ & 3 & 2 \\
\hline
\end{tabular}

Fuente: Autoras.

\section{Red semántica de información}

Se exploró el discurso que tienen los adultos mayores respecto al envejecimiento y la institución, considerando que la primera es una categoría pertinente que hace parte de ese proceso dinámico que sucede a lo largo del ciclo vital, por lo que existe una relación e influencia directa sobre la vejez. Esto lleva a entender el envejecimiento como ese proceso dinámico sobre el cual existe una gran variedad de cambios físicos, cognitivos y emocionales que son progresivos, lo que se refleja durante la etapa de vejez.

De acuerdo con los resultados presentados en la Figura 3 , el envejecimiento se asocia con aquellos aspectos positivos y hobbies que funcionan como una forma de compensar, fortalecer y mejorar la eficacia de los procesos cognitivos, físicos o motores, a fin de que el adulto mayor pueda restablecer ciertas funciones alteradas

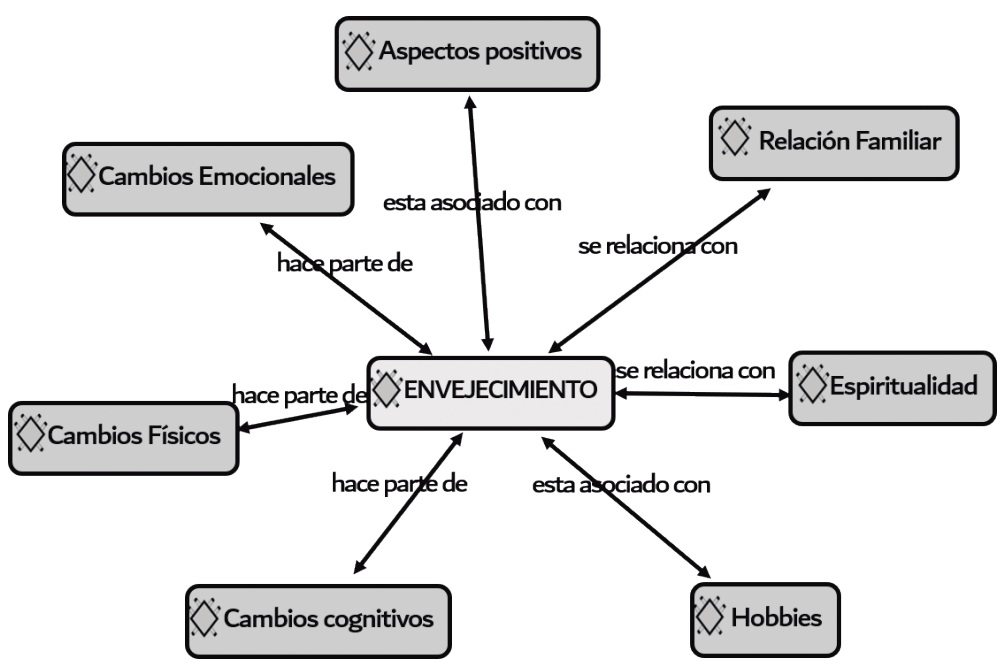

Figura 3. Subcategoría envejecimiento. Geriátrico privado. Fuente: Autoras.

o deterioradas durante el proceso de envejecimiento. Con respecto a los cambios emocionales, mencionan que la lectura y el trato recibido por el personal de la institución los lleva a sentirse felices y tranquilos; así lo refiere P5: "Ay, yo me siento feliz, me siento bien, yo soy alegre, soy feliz, la vida me ha tratado bien". Por otro lado, la espiritualidad y la relación familiar hacen parte de este proceso, $y$, a medida que se va envejeciendo, se fortalecen esos lazos espirituales, sociales y familiares que bridan al adulto mayor un estado de confort, tal cual como lo menciona P1: "ser servicial y mostrarle al señor, pero con hechos, mas no con palabras"; y, a su vez, P5: "Ay no, yo he encontrado al envejecer y todo buenas amistades..., uno no puede decir amigos si no son contados en los dedos".
Desde una perspectiva muy diferente, los gerontes del establecimiento público se expresaron con respecto a la categoría de envejecimiento. En la Figura 4, se aprecia que el envejecimiento se relaciona con un deterioro físico y cognitivo que conduce a una limitación del movimiento, el desplazamiento y la agilidad, tal como lo verbalizan PJ2: "Arrugas, poca agilidad, el hablar con dificultad"; PJ5: "arruga, el pelo canoso, ya uno no puede salir solo, ya no oye bien, ni ve bien". Según ellos, estos aspectos se asocian con las relaciones familiares quebrantadas, que afectan directamente el estado emocional del adulto mayor, lo que genera en algunas emociones de rabia, tristeza y dolor, y pensamientos suicidas por la soledad y el abandono. PJ3: "Se entristece uno por todo, y a veces le da unas arrancadas de rabia y pelea uno con todo el mundo aquí". PJ1: "Triste porque uno solo por aquí". PJ4: "Emocionalmente me siento solo". 


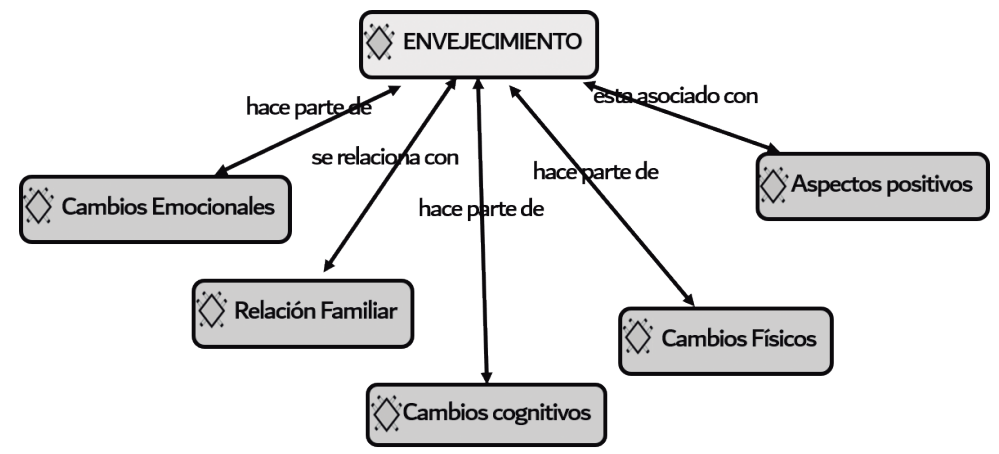

Figura 4. Subcategoría envejecimiento. Geriátrico Público. Fuente: Autoras.

\section{b) Análisis comparativo de la perspectiva discursiva \\ (geriátrico privado frente a geriátrico público)}

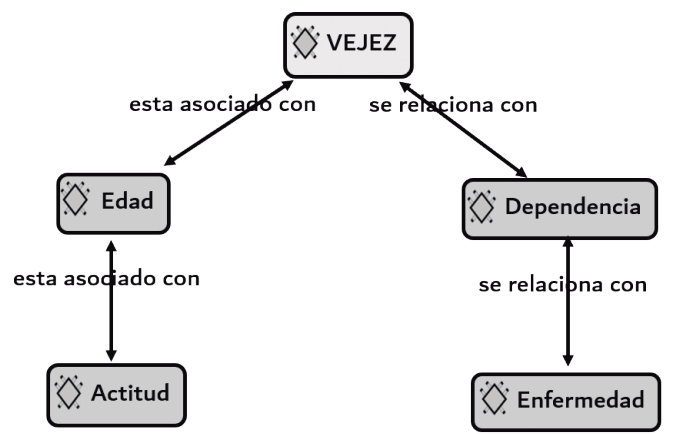

Figura 5. Subcategoría Vejez. Geriátrico Privado. Fuente: Autoras.

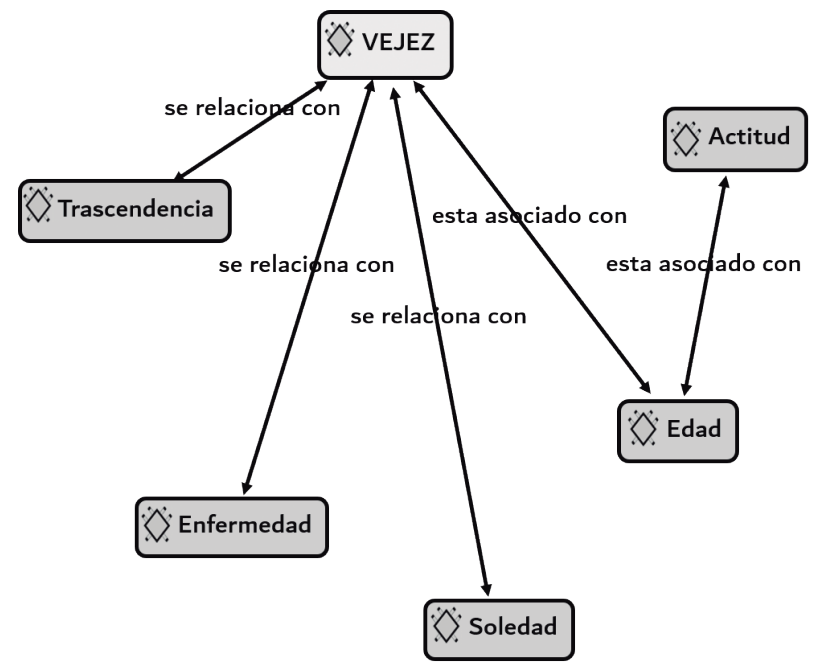

Figura 6. Subcategoría Vejez. Geriátrico Público. Fuente: Autoras.

En las Figuras 5 y 6 se puede evidenciar que, respecto al geriátrico público, la edad es considerada por los adultos mayores como el punto de partida de la vejez. Se acompaña de una percepción que hace parte de un proceso indisoluble de la vida, pero que viene dada por enfermedades, soledad, abandono y necesidad de compañía. Pasa lo contrario con los adultos mayores del geriátrico privado, que consideran que la edad no determina la vejez; es decir, para ellos la vejez es cuestión
1) Vejez

. Enfermedad 
Laura Juliana Barchelot Aceros, Lina Katherine Valencia Silva, Suzette Tatiana Poveda Esteban, Emily Carolina González Clemente Diferencias en las perspectivas discursivas sobre la vejez y la muerte en adultos mayores pertenecientes a dos instituciones geriátricas de Bucaramanga

aspecto familiar, laboral y de estudio; por ejemplo, mi poliomielitis, eso es de hace mucho, no por la vejez.

Hay una diferencia en la forma de ver la vejez, pues los gerontes del geriátrico privado tienen una perspectiva positiva respecto a esta. La asumen como un proceso obvio y natural, del cual hay que tomar conciencia e interiorizar. Es preciso prepararse de cara a los cambios que esta involucra, como los cambios físicos y cognitivos.

Caso contrario ocurre en el geriátrico público, donde la perspectiva que prevalece frente al envejecimiento y la vejez es negativa, dado que los asocian con una idea de deterioro y muerte que despierta en ellos miedo, y, por lo tanto, se genera una tendencia a negar el proceso evolutivo natural a envejecer. PJ2: "No he encontrado nada, he estado enfermo, he encontrado negativo porque desde hace 7 años era una persona activa, he bajado la guardia". PJ1: "Positivo eso que, nada, no, nada, eso uno enfermo, cada vez es más". Atribuyen a la vejez la soledad y las enfermedades, y estas últimas son consideradas como un factor incapacitante o limitante.

Otro aspecto importante, y que marca una diferencia en cómo se percibe la vejez, es la familia (red de apoyo), que, como se ha venido mencionando, hace parte esencial del proceso de adaptación e interiorización de los cambios que involucra la vejez. Esta se percibe, por su parte, como aquella etapa en la cual se encuentran abandonados, donde dejan de ser importantes para la familia. Se encontró que en el geriátrico público las relaciones familiares de los adultos mayores que hicieron parte de la investigación son significativamente escasas y deterioradas. PJ5: "Yo estoy sola, no tengo a nadie". PJ4: "a uno ya no le dedican tiempo, le da a uno sentimiento, ya no es como en aquellos tiempos..., ahorita ya no hay familia".
Consideran la vejez como una etapa de la vida en la cual se encuentran solos y desamparados, lo que afecta su estado de ánimo, y los conduce a un estado depresivo en que buscan acabar con ese sentimiento de soledad. PJ4: "Si hay un señor, yo me pongo a conversar con él, y él dice que quiere pegarse un tiro, y así estoy yo, pero esperamos que venga yo, y tenemos resistencia, entonces cuando yo entré aquí él me dio mucho ánimo".

A diferencia del geriátrico privado, donde los lazos de las relaciones familiares suelen ser positivos. P3: "Magníica, en mis oraciones le pido al señor que los llene de tolerancia y convivencia familiar".

Se considera también importante resaltar el significado de trascendencia que el adulto mayor del geriátrico público le otorga a la vejez, lo que no se evidenció en el discurso de los adultos mayores del geriátrico privado. La trascendida, de acuerdo con Seelbach (2013), se da durante esta etapa de la vida, donde el adulto mayor se preocupa por alcanzar la autorrealización, por medio del legado que deja. PJ4: "Dejar un legado bueno a mis hijos, nietos, eso de no cometer los errores que uno cometió en la juventud, que salgan y que luchen".

Por lo tanto, se considera a la vejez una etapa evolutiva que involucra aspectos que, de una u otra manera, pueden afectar la percepción que el geronte se hace respecto a ella. Esto se evidencia principalmente en las redes de apoyo, que, si bien son importantes en todas las etapas de la vida, durante la vejez juegan un papel esencial para el adulto mayor. Al no recibir este apoyo, se pueden presentar estados de ánimo que afectan el bienestar y equilibrio emocional del geronte, y, por ende, la percepción que este tiene respecto a la forma de asumir la vejez (positiva o negativamente).

\section{2) Muerte}

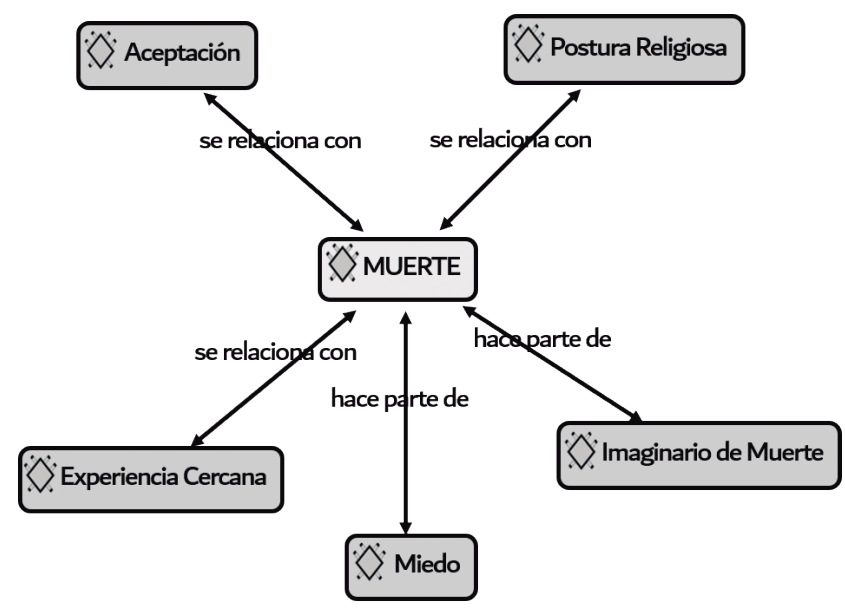

Figura 7. Subcategoría Muerte. Geriátrico privado. Fuente: Autoras. 


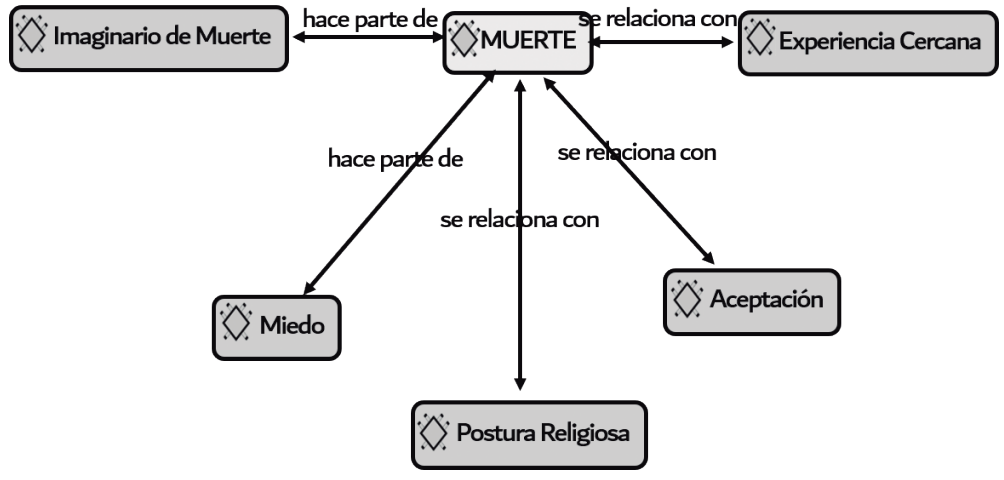

Figura 8. Subcategoría Muerte. Geriátrico público. Fuente: Autoras.

La diferencia que se encuentra en los geriátricos (uno público y uno privado) respecto a la muerte (ver Figuras 7 y 8) radica en que un poco más de la mitad de la población percibe la muerte como un proceso natural, que hace parte del ser humano y que es impredecible. Los adultos del geriátrico privado evidenciaron una postura ceñida a lo religioso. La muerte se asocia con un proceso inevitable que conducirá al encuentro con Dios; sin embargo, este discurso es reforzado por el distanciamiento de los hijos, la separación o muerte del cónyuge y la jubilación, tal como lo evidencian P2: "Para mí mejor que me muera, es un deseo, ya uno solo". P3: "Una frustración porque vivo enamorado de la vida, moriría resentido con el señor". Y se suma a ello un factor de religiosidad: P2: "Entregarle cuentas al señor, que él ya quiere llevar a uno"; P1: "Es ir al encuentro con el señor".

De igual forma, en el geriátrico público también se genera una serie de imaginarios con respecto a la muerte, asociados a sentimientos positivos fundamentados en posturas religiosas. La aceptación de esta etapa se caracteriza, a su vez, por un discurso tranquilo y seguro. PJ3 expresa: "Alegría, sería un regalo porque así uno no sufre". Para PJ4: "Alegría, yo deseo morirme"; y también resaltan posturas religiosas. PJ3: "Es un regalo, porque es un encuentro con Dios".

\section{Miedo y frustración}

PJ1: "Miedo, porque la muerte no avisa". P3 relata: "Una frustración, porque vivo enamorado de la vida, moriría resentido con el señor". Y P5: "Miedo, tristeza, la vida es muy linda y no quisiera morir tan pronto".

Cabe destacar que, respecto al imaginario de muerte, no existe diferencia en la forma en que perciben este factor. Al contrario, los discursos son similares, dado que manifiestan el deseo de una muerte súbita, en la que no haya sufrimiento. PJ3 expresa: "Algo repentino, que uno no sufra". PJ4: "Yo quiero acostarme y no levantarme". PJ2: "sin sufrir, algo rápido". PJ5: "yo quiero morirme de sopetón". P3: "algo súbito". P5: "yo quisiera morirme aquí sentada, sola".
Entonces, es evidente que, al hablar de la muerte con los adultos mayores, se generan diversos sentimientos, que en su mayoría se fundamentan con una postura religiosa. Esto genera congruencia con un discurso tranquilo y seguro, como respuesta a una aceptación sobre esta etapa, pero sin dejar de lado ese sentimiento natural que se asocia a esta etapa de la vida: el miedo o la negación, algo completamente natural, y que, con relación al discurso de algunos adultos mayores, es producto de múltiples factores, como, por ejemplo, el distanciamiento que han tenido los hijos, la separación y la jubilación, entre otros.

\section{Conclusiones}

Teniendo en cuenta la perspectiva fenomenológica del discurso de los adultos mayores institucionalizados de los hogares geriátricos privado y público de Bucaramanga, y de cada una de las categorías abordadas, así como los aspectos más predominantes que surgieron de la investigación en relación con la percepción discursiva del adulto mayor frente a la vejez y la muerte, se concluye que:

- En el hogar geriátrico público los adultos mayores no cuentan con redes de apoyo consolidadas y funcionales. Se considera que las relaciones con la familia son escasas o deterioradas, situación que genera en los adultos diferentes alteraciones emocionales como tristeza, dolor, amargura y aburrimiento. Estos aspectos se asocian con la presencia de sentimientos de soledad y abandono, lo que genera un mayor riesgo de desarrollo de síntomas depresivos. Sumado a ello, las dinámicas de relación entre los adultos mayores que allí se encuentran internados se caracterizan por la conflictividad y la escaza vinculación afectiva entre ellos mismos. Por ende, se hace necesario la consolidación de redes de apoyo institucionales, en aras de la prevención de las alteraciones del estado de ánimo que podrían ser concomitantes con un deterioro cognitivo.

- Se encontró dentro del discurso respecto a la muerte de los adultos mayores del hogar geriátrico público pensamientos e ideaciones suicidas, demarcados por el 
Laura Juliana Barchelot Aceros, Lina Katherine Valencia Silva, Suzette Tatiana Poveda Esteban, Emily Carolina González Clemente Diferencias en las perspectivas discursivas sobre la vejez y la muerte en adultos mayores pertenecientes a dos instituciones geriátricas de

Bucaramanga

mismo contexto en el cual se encuentran, los cambios que involucran la vejez, la minusvalía y, claro está, los aspectos anteriormente mencionados.

- En el hogar geriátrico privado el adulto mayor cuenta con ciertos hobbies que le permite mantenerse activo, con lo que ocupan el tiempo en actividades que le son satisfactorias. En consecuencia, asume los cambios que trae el envejecimiento con mayor naturalidad; entre estos, aquellas limitaciones físicas producto de la enfermedad que lo aqueja, que asume como circunstancias ajenas o independientes a la edad. Es decir, mantiene una postura tranquila, dado que cuenta con unas redes de apoyo externas (familiares y amigos) e internas, a nivel de la institución, que lo fortalece y ayuda a ver la vejez desde una óptica o perspectiva positiva.

- En ambas instituciones geriátricas prevalece en mayor grado una postura religiosa que influye en la percepción de los adultos mayores frente a la muerte, al considerar esta como una intervención divina, en la que Dios es quien define cómo morirán y lo que suceda después de la muerte. Esto es, se apoyan en la religión y en sus creencias como una forma de mitigar el miedo hacia la muerte; adquieren fortaleza a través de su fe, lo que se refleja en los hábitos que practican (oraciones). Construyen un significado de la muerte basada en la relación que tienen con Dios, así como en las experiencias cercanas. A partir de esto, construyen también un imaginario de muerte sobre el que prevalece el morir de manera súbita y tranquila.

- El trato del cuidador hacia el adulto mayor juega un papel importante en la percepción que el geronte se hace de sí mismo en relación con la vejez y la muerte. Estos conceptos se asocian al cuidado que ellos brindan y al mensaje que transmiten hacia el adulto mayor. Por esta razón, con base en el discurso de los gerontes del geriátrico privado, los cuidadores en este hogar generan comentarios que aluden a una falta de respeto hacia la condición de anciano que estos poseen. Esto quizá se atribuye a la carga laborar, a la falta de preparación o a la capacitación.

- Es importante que las instituciones geriátricas consideren integrar más activamente la familia como centro o red de apoyo emocional para los adultos mayores institucionalizados, pues, como se evidenció en la narrativa de los gerontes, la percepción y aceptación que ellos otorgan al envejecimiento, la vejez y la muerte se enlazan con la familia. Esta representa el soporte emocional más importante, pero, así mismo, juegan un papel crucial el personal que hace parte de la institución geriátrica, la dinámica de la misma institución y la relación con los demás adultos mayores. De este modo, los gerontes podrán interiorizar el desenlace de la vida con mayor naturalidad.

\section{Referencias}

Aranda, C., Pando, M., Flores, M. y García, T. (2001). Depresión y redes sociales de apoyo en el adulto mayor institucionalizado de la zona metropolitana de Guadalajara, Jalisco. Revista Psiquiatría Facultad de Medicina Barna, 28(2), 70.

Barenys, M. P. (2012). Los geriátricos, de la institucionalización al extrañamiento. Revista Kairós: Gerontologia, 15(4), 7-24.

Cardona-Arango, D., Estrada-Restrepo, A., ChavarriagaMaya, L. M., Segura-Cardona, Á. M., Ordoñez-Molina, J. y Osorio-Gómez, J. J. (2010). Apoyo social dignificante del adulto mayor institucionalizado. Medellín, 2008. Revista de Salud Pública, 12(3), 414-424.

Dulcey, E. (2015). Envejecimiento y vejez: Categorías y conceptos. Fundación Cepsiger para el desarrollo humano.

Edwards, D. (1997). Discourse and Cognition. Sage Publications.

Erikson, E. (1982). The Life Cycle Completed. Norton.

Garay, A., Iñiguez, L. y Martínez, L. M. (2005). La perspectiva discursiva en psicología social. Subjetividad y Procesos Cognitivos, (7), 105-130.

Garrido, A. y Álvaro, J. L. (2007). Psicología social. Perspectivas psicológicas y sociológicas. McGraw Hill.

Gergen, K. J. (1999). An Invitation to Social Construction. Sage Publications Itda.

Gergen, K. J. (2007). Construccionismo social - aportes para el debate y la práctica. Ediciones Uniandes.

Hernández, R., Fernández, C. y Baptista, M. (2014). Metodología de la investigación. http://observatorio. epacartagena.gov.co/wp-content/uploads/2017/08/ metodologia-de-la-investigacion-sexta-edicion. compressed.pdf

López, A. M. (2009). ¿Qué es el envejecimiento? El envejecimiento, su origen, su transcurrir y sus secuelas es un tema que nos preocupa a todos. https://www. intramed.net/contenidover.asp?contenidolD $=58673$

López, M. y Marín, R. (2016). Revisión teórica y empírica desde la psicología sobre representaciones sociales del envejecimiento y la vejez en Latinoamérica y España (2009-2013). Revista Científica General José María Córdova, 4(17), 155-202.

Ministerio de Salud y Protección Social [MinSalud]. (2013). Envejecimiento demográfico. Colombia 1951-2020. Dinámica demográfica y estructuras poblacionales. https://www.minsalud.gov.co/sites/rid/Lists/ Biblioteca Digital/RIDE/DE/PS/Envejecimiento-demograficoColombia-1951-2020.pdf

Ministerio de Salud y Protección Social [MinSalud]. (2018). Envejecimiento y Vejez. https://www.minsalud.gov. co/proteccionsocial/promocion-social/Paginas/ envejecimiento-vejez.aspx

Ollar, J. A. (1997). Envejecimiento y función cognitiva. De la declinación al deterioro. En C. A. Mangone, R. F. Allegri, R. L. Arizaga y J. A. Ollari (eds.), Demencia: 
enfoque multidisciplinario. (pp. 71-97). Sagitario Editores.

Organización Mundial de La Salud [OMS]. (2017). La salud mental y los adultos mayores. https://www.who.int/ es/news-room/fact-sheets/detail/la-salud-mental-ylos-adultos-mayores

Río, K., Dominguez, P. y Pignocchi, A. (2014). Evaluación del capital psíquico en adultos mayores auto-válidos y dependientes que residen en la ciudad de Mar del Plata [tesis de grado, Universidad Nacional de Mar del Plata). Repositorio RPsico: http://rpsico.mdp.edu.ar/ handle/123456789/50

Rivera, J. y Mancinas, S. (2007). El anciano ante la muerte: Análisis del discurso en el noreste de México. Estudios Sociológicos, 15(74), 341-367.

Rodríguez, G., Gil, J. y García, E. (1999). Metodología de la investigación cualitativa. (Segunda ed). Aljibe.

Rubio, R. (1981). El problema de la muerte en la tercera edad desde la perspectiva psicológica. Revista de Psicología General y Aplicada, 36(4), 719-727.

Santos, M. y Valencia, N. (2015). Envejecer en Colombia. Red de Revistas Científicas de América Latina, El Caribe, España y Portugal, 71, 61-81.

Seelbach, G. (2013). Teorías de la personalidad (1 ra Edició). http://www.aliat.org.mx/BibliotecasDigitales/ Psicologia/Teorias_de_la_personalidad.pdf

Serra, E.y Abengózar, M. (1990). Ancianidad y preparación para la muerte. Anales de Psicología, 6(2), 147-158.

Vargas-Jiménez, I. (2012). La entrevista en la investigación cualitativa: nuevas tendencias y retos. Revista Electrónica Calidad en la Educación Superior, 3(1), 119-139.

Zavala, M., Vidal, D., Castro, M., Quiroga, P. y Klassen, G. (2006). Funcionamiento social del adulto mayor. Ciencia y Enfermería, 12(2), 53-62. 